\title{
Aplicação de programa de análise de imagens na interpretação de fotomicrografias de alta resolução de argilominerais
}

\section{(Application of image analysis software to interpretation of high resolution photomicrographs of clay minerals)}

\author{
M. P. M. A. Baroni ${ }^{1}$, S. R Teixeira ${ }^{2}$, J. B. Dixon ${ }^{3}$, G. N. White ${ }^{3}$ \\ ${ }^{1}$ Laboratório Associado de Computação e Matemática Aplicada, Instituto de Pesquisas Espaciais - INPE \\ S. José dos Campos, SP, Brasil 12227-010 \\ ${ }^{2}$ Departamento de Física Química e Biologia, Universidade Estadual Paulista - UNESP \\ C.P. 467, Presidente Prudente, SP, Brasil 19060-000. \\ ${ }^{3}$ Soil \& Crop Sciences Dept., Texas A \& M University, College Station, TX, USA 77843-2474 \\ rainho@prudente.unesp.br
}

\begin{abstract}
Resumo
O objetivo principal deste trabalho é destacar o potencial da análise de imagens na interpretação de fotomicrografias de argilominerais. Foram usadas imagens de argilas obtidas com um microscópio eletrônico de transmissão de alta resolução e um programa de análise de imagens. A manipulação das imagens, usando as diferentes ferramentas do programa, permite melhorar sua qualidade e quantificar alguns parâmetros importantes, tais como a morfologia estrutural dos diferentes compostos, os espaçamentos interplanares e o ângulo de inclinação das franjas de rede, o tamanho e a morfologia de grãos do material, dentre outras medidas de interesse. Foram aplicados diversos filtros para melhorar a imagem inicial e a transformada rápida de Fourier foi aplicada em imagens individuais de grãos que apresentam franjas de rede. Além desses filtros, o programa tem outras ferramentas que podem ser usadas para fazer medidas (de diâmetros, áreas, comprimentos etc.), que permitem determinar distâncias interplanares dos planos (hkl) selecionados. Embora o programa possa ser usado em outras aplicações interessantes (por exemplo, em análise metalográfica de fotografias obtidas com microscópio óptico), neste trabalho são mostrados os resultados obtidos na análise de algumas franjas de rede de argilominerais.

Palavras-chave: análise de imagens, argilas, microscopia de alta resolução.
\end{abstract}

\begin{abstract}
The main objective of this paper is to show the potential image analysis on microscopy images interpretation of clay minerals. An image analysis software was applied to manipulate High-Resolution Transmission Electron Microscopy images of clays to improve them. Afterwards some parameters such as interplanar spacing were measured. It is also possible to measure others parameters like the angle between crossed lattice fringes, grain morphology, orientation, elongation, and crystallite sizes. The technique involves the application of fast Fourier transforms to single particles, application of the spike-boost program accessory to improve the images, and after that it is applied the inverse FFT. Although the program can be used to other interesting applications (for example, metallographic analysis of optical photomicrographs) in this paper the results obtained from the analysis of some clays minerals lattice fringes are shown.
\end{abstract}

Keywords: image analysis, clays, high resolution microscopy.

\section{INTRODUÇÃO}

Um dos meios auxiliares mais úteis para compreender a estrutura dos materiais é a observação de sua imagem usando um microscópio óptico ou eletrônico. Uma fotomicrografia desse tipo revela detalhes interessantes da microestrutura não visíveis a olho nu. Também, podem ser obtidas informações quantitativas sobre características estruturais do material como, por exemplo, distância interplanar, orientação, distribuição de franjas de rede, tamanhos e diâmetros.

Imagens de microscopia eletrônica de transmissão de alta resolução (MET-AR), obtidas com ótimas condições de orientação e focagem, mostram a estrutura cristalina com clareza distinguindo as áreas escuras, que correspondem às regiões de alta densidade atômica, das áreas claras, de baixa densidade atômica como por exemplo, os túneis estruturais. Por outro lado quando as imagens não são boas, muitas informações podem ser perdidas. Essas imagens, bem como 
as consideradas boas, podem ser melhoradas usando um programa de análise de imagens. Em geral, esses programas apresentam muitas ferramentas que permitem uma melhor visualização das imagens [1]. A aplicação de análise de imagens em microscopia eletrônica de alta resolução tem sido bastante aplicada em várias áreas das ciências. Como exemplo podem ser citados os estudos, de nanocristais em materiais amorfos, ou parcialmente orientados [2], em fuligem e negro de fumo [3], de estruturas de cerâmicas supercondutoras [4], dentre outros.

O presente trabalho aplica um programa de análise de imagens em fotomicrografias de argilas que constituem a fração mais fina dos solos. Imagens de nanopartículas desses minerais obtidas usando MET-AR foram analisadas usando o programa Image PRO-PLUS. Franjas de rede e defeitos estruturais, foram avaliados, fornecendo um ótimo exemplo de aplicação desse programa no estudo de argilominerais.

Este trabalho destaca o potencial de aplicação de um sistema modular de análise de imagens para obtenção de informações morfológicas e estruturais de fotomicrografias de argilominerais, obtidas com microscopia eletrônica de transmissão de alta resolução.

\section{MATERIAIS E MÉTODOS}

\section{Obtenção das fotomicrografias}

As imagens de argilas, de alguns solos da região de Presidente Prudente, SP, foram obtidas usando, dentre outras técnicas, microscopia eletrônica de transmissão de alta resolução (MET-AR) [5-7].

A preparação das amostras e as imagens foram feitas no Laboratório de Mineralogia de Solos e no Centro de Microscopia Eletrônica da Texas A \& M University (TAMU), em College Station, Texas, EUA. Foi usado um microscópio eletrônico de transmissão JEOL 2010 TEM, operado com $200 \mathrm{kV}$, para obtenção das imagens de alta resolução. Em muitas destas fotomicrografias são observadas franjas de rede e grãos de argilas com variadas morfologias: acicular, tabular, placa, fibra [8].

As imagens analisadas foram obtidas da fração argila de um solo da região de Presidente Prudente, no oeste do Estado de S. Paulo. Nestas imagens são observadas raias denominadas "lattice fringes" (franjas de rede), que são imagens formadas pelos planos de átomos da rede cristalina do material analisado (planos com índice de Miller (hkl)). Usando duas lupas, uma comum e uma com escala (aumento de $10 \mathrm{X}$ ), foram selecionadas as áreas a serem analisadas. A partir de agora, a palavra imagem significa a área selecionada na fotomicrografia.

\section{Metodologia para a obtenção das imagens}

As fotomicrografias foram digitalizadas usando um scanner padrão. Cada imagem selecionada foi analisada no laboratório de microscopia óptica do Departamento de Física, Química e Biologia da Faculdade de Ciências e Tecnologia - Unesp, Presidente Prudente. Este laboratório está equipado com microscópio Leica DMRX com um computador Pentium III e um sistema de digitalização de imagens. Além dos softwares que acompanham o equipamento, este sistema dispõe do programa Image-Pro Plus 4.0, da Media Cybernetics. O Image-Pro Plus 4.0 tem vários subprogramas e modos para armazenar as medições ou outros tipos de informação, para cada aplicação específica, mas que trabalham integrados dentro do programa principal. Foram aplicados diversos filtros para melhorar a imagem e estudar a morfologia da superfície das amostras. Devido a sua periodicidade, as franjas de rede foram analisadas usando a Transformada Rápida de Fourier (FFT) de modo a realçar as franjas e os defeitos estruturais da rede cristalina e melhor visualiza-las.

Primeiro é aplicada a FFT na área selecionada (franjas da rede direta), que fornece uma imagem chamada de espectro de frequiência (rede recíproca), com pontos distribuídos simetricamente ao redor da origem dos eixos xy. Cada par destes pontos equivale a um conjunto de franjas de rede com mesmo espaçamento interplanar d. As posições destes pontos (distância aos eixos) estão relacionadas com o valor do espaçamento interplanar e com o ângulo de orientação das franjas, em relação aos eixos. O espectro de freqüência é semelhante à imagem de difração de elétrons (rede recíproca) que seria obtida para um conjunto de planos (hkl) da rede cristalina. Foi aplicada a ferramenta spike-boost em cada ponto do espectro de frequiência da imagem, para aumentar o contraste deles com relação ao fundo da imagem. Ao fazer a transformada inversa (IFFT), a imagem inicial das franjas é recuperada com maior nitidez, permitindo melhor visualização das franjas de rede, de seus defeitos e, consequentemente, determinar seus valores. As distâncias interplanares foram medidas usando como referência a barra de calibração da fotomicrografia.

\section{A transformada de Fourier e a rede recíproca}

A rede recíproca é uma rede tri-periódica derivada das redes de Bravais (rede direta). Ela permite resolver mais rapidamente certos problemas de cristalografia geométrica e, ainda, enunciar de uma maneira mais simples certos aspectos da difração de raios $\mathrm{X}$ pelos cristais. As redes recíprocas diferem das redes de Bravais apenas pelas suas dimensões absolutas e, portanto, podem ser consideradas como sendo uma única rede, representadas em diferentes escalas. Numa rede direta os átomos de um cristal podem ser posicionados sobre planos, representados por índices (hkl), definidos em relação a um sistema de coordenadas (xyz). Ao incidir um feixe de elétrons (ou raios X) sobre esse cristal, ele será espalhado em diversas direções, de acordo com a Lei de Bragg e com o plano (hkl) que espalha o feixe de radiação. Cada conjunto de planos (hkl) gerará um ponto, 
representando a posição geométrica daquele conjunto de planos no cristal. O conjunto de pontos gerados, para cada conjunto de planos, resultará numa nova rede denominada de rede recíproca. Portanto, cada um dos nós do espaço recíproco corresponde a um conjunto de planos da rede cristalina $[9,10]$. A posição dos nós, pontos onde os três planos que formam a rede direta se cruzam, é representada por um vetor $\mathbf{r}=\mathrm{u} \mathbf{a}+\mathrm{vb}+\mathrm{w} \mathbf{c}$, onde $(\mathrm{u}, \mathrm{v}, \mathrm{w})$ são inteiros que definem as coordenadas do ponto na extremidade do vetor $\mathbf{r}$. A posição dos nós da rede recíproca será definida por um vetor $\mathrm{r}_{\mathrm{hkl}}^{*}=\mathrm{h} \mathbf{a}^{*}+\mathrm{k} \mathbf{b}^{*}+\mathrm{l} \mathbf{c}^{*}$, onde $(\mathrm{h}, \mathrm{k}, \mathrm{l})$ são inteiros. Colocando os eixos da rede recíproca, normais aos planos (definidos por pares de eixos) da rede direta $\left(\mathbf{a}^{*} \perp \mathbf{b}\right.$ e $\mathbf{c}$, $\mathbf{b}^{*} \perp \mathbf{a}$ e $\mathbf{c}, \mathbf{c}^{*} \perp \mathbf{a}$ e $\left.\mathbf{b}\right)$ pode-se definir a rede recíproca diretamente das equações:

$$
\begin{array}{lll}
\mathbf{a}^{*} \cdot \mathbf{a}=1 & \mathbf{b}^{*} \cdot \mathbf{a}=0 & \mathbf{c}^{*} \cdot \mathbf{a}=0 \\
\mathbf{a}^{*} \cdot \mathbf{b}=0 & \mathbf{b}^{*} \cdot \mathbf{b}=1 & \mathbf{c}^{*} \cdot \mathbf{b}=0 \\
\mathbf{a}^{*} \cdot \mathbf{c}=0 & \mathbf{b}^{*} \cdot \mathbf{c}=0 & \mathbf{c}^{*} \cdot \mathbf{c}=1
\end{array}
$$

onde $\mathbf{a}, \mathbf{b}$ e $\mathbf{c}$ são vetores da rede cristalina (direta) e $\mathbf{a}^{*}, \mathbf{b}^{*}$ e c* são vetores da rede recíproca.

Neste caso, onde os eixos das duas redes são perpendiculares entre si, os eixos da rede recíproca terão comprimentos que são o recíproco dos comprimentos dos eixos da rede cristalina (direta). O espaçamento $d_{\mathrm{hkl}}$ entre os planos (hkl) da rede direta, em qualquer sistema cristalino, será o recíproco do comprimento do vetor $\mathrm{r}_{\mathrm{hk}}^{*}{ }^{*}$

$$
\mathrm{r}_{\mathrm{hkl}}^{*}=\frac{1}{\mathrm{~d}_{\mathrm{hkl}}}
$$

Este valor poderá ser obtido, medindo-se a distância dos pontos da rede recíproca ao centro dos eixos de referência na figura (espectro de frequiências) $[9,10]$.

Portanto, o que será feito com o programa de análise de imagens é: (1) transformar a rede direta em rede recíproca, usando a transformada de Fourier (FFT), (2) intensificar o brilho de cada ponto da rede recíproca usando a ferramenta "spike boost" e (3) aplicar a transformada inversa de Fourier (IFFT) para recuperar a rede direta, agora com melhor nitidez. Esta imagem melhorada, além da medida de $d_{\mathrm{hkl}}$, também permitirá medir os ângulos entre planos (hkl) que se cruzam.

\section{RESULTADOS E DISCUSSÃO}

\section{Análise das imagens}

A aplicação de Transformada de Fourier Rápida (FFT) forneceu resultados excelentes, melhorando a imagem e permitindo observar com maior nitidez as franjas e defeitos (por exemplo, deslocação em cunha) de rede.

A Fig. 1 mostra uma fotomicrografia da qual foram selecionadas duas áreas (1 e 2) para aplicação do programa

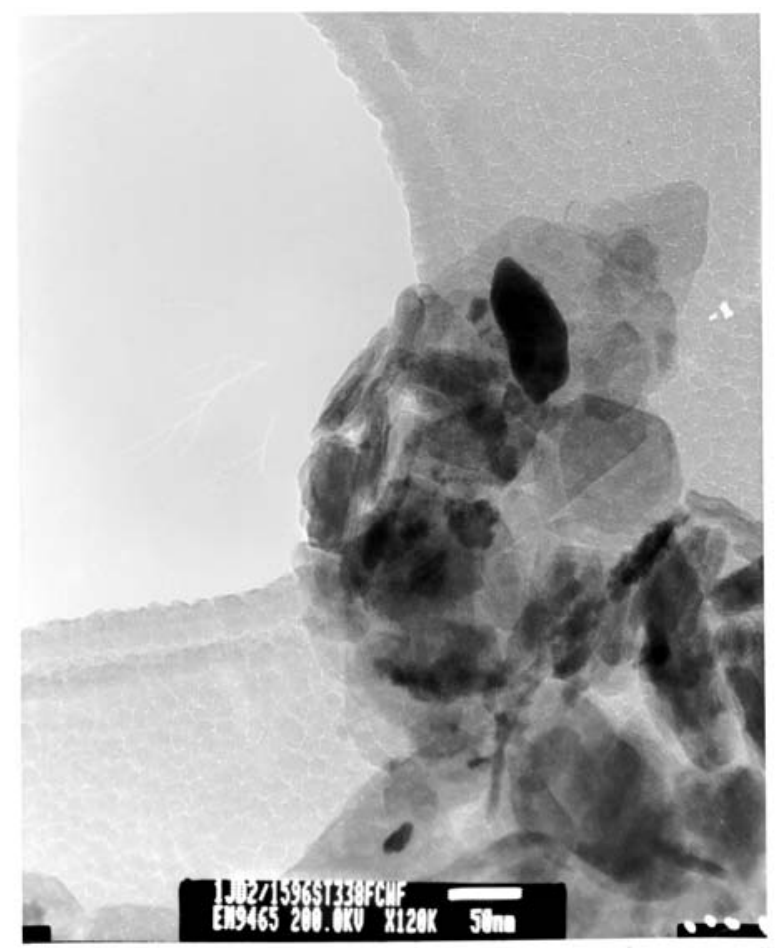

Figura 1: Fotomicrografia mostrando duas áreas selecionadas. A morfologia e a cor do grão na área 1 , indicam a presença de óxido de ferro. A área 2 apresenta uma estrutura longa (ripa) e fina, como uma argilomineral.

[Figure 1: Photomicrography showing two selected areas. In area 1 , the grain morphology and color indicate iron oxide. Area 2 shows a long and thin structure (lath) like a clay mineral.]

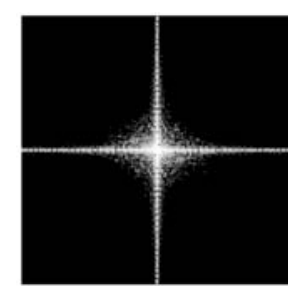

(a)

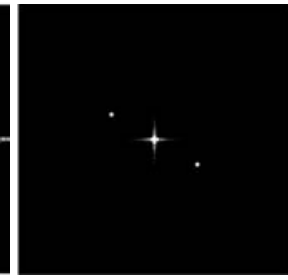

(b)

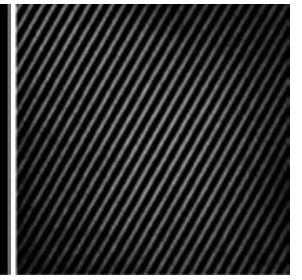

(c)
Figura 2: Análise da Área 1, (a) espectro de freqüência obtido aplicando FFT e usando ferramentas de contraste, (b) espectro de freqüência após aplicar nove vezes a ferramenta spike boost para aumentar brilho do ponto, (c) área 1 recuperada (IFFT) do espectro anterior, mostrando as franjas de rede usadas para medir o $\mathrm{d}_{\mathrm{hkl}}$ desses planos.

[Figure 2: Analysis of area 1, (a) frequency spectrum obtained using FFT and contrast tools, (b) frequency spectrum after nine applications of the spike boost tool to increase the point brightness, (c) area 1 recovered (IFFT) from (b) showing the lattice fringes used to measure $d_{h k l}$ values.]

de análise de imagem.

A Fig. 2 mostra o resultado da aplicação de diversas ferramentas do programa na área 1 da Fig. 1. A Fig. 2a mostra o espectro de frequiências melhorado usando ferramentas de contraste. Pode-se observar (Fig. 2c) que esta área do grão 
não apresentou nenhum tipo de defeito estrutural. $\mathrm{O}$ valor médio obtido para a distância interplanar deste grão é 0,89 nm ( 9 A). Este valor indica argilomineral do grupo da mica. Entretanto, devido à morfologia e a cor escura do grão (maior absorção do feixe de elétrons), este valor corresponde à distância interplanar a da cela unitária da magnetita $\left(\mathrm{Fe}_{3} \mathrm{O}_{4}\right)$, que mede $0,839 \mathrm{~nm}$. Em outro trabalho [7], usando outras técnicas analíticas, foi observado que este óxido de ferro é comum neste tipo de solo.

A Fig. 3 mostra o resultado da análise da área 2, que foi escolhida por apresentar muitas franjas de rede. Entretanto, quando foi aplicado a FFT o espectro de frequiências obtido não apresentou os nós das franjas de rede tendo sido necessário melhora-lo usando a ferramenta de aumento de contraste. No espectro melhorado foram observados dois conjuntos de pontos simétricos. Ambos apresentaram distâncias interplanares próximas a 0,9 nm. Novamente, estes valores indicam argilominerais do grupo da mica, comum nesses solos [5]. A área não apresentou nenhum tipo de defeito de estrutura e o valor médio do ângulo entre esses dois conjuntos de franjas é de $98,91^{\circ}$.

\section{Ponto 1}

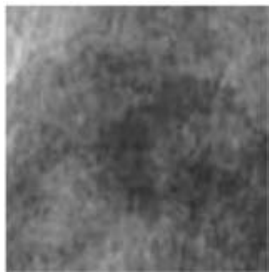

(a)

Ponto 2

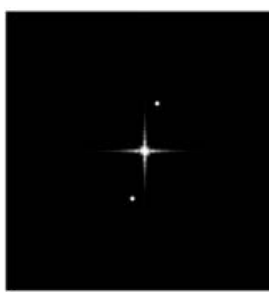

(d)

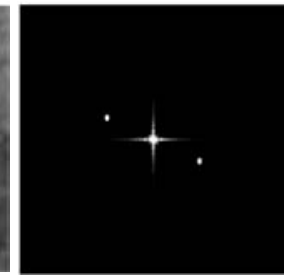

(b)

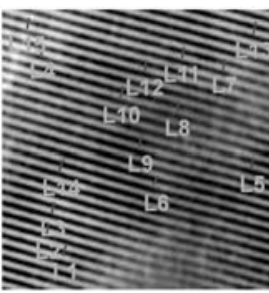

(e)

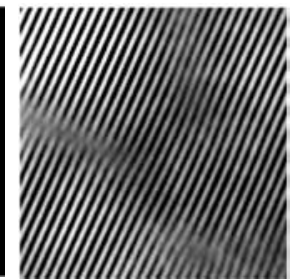

(c)

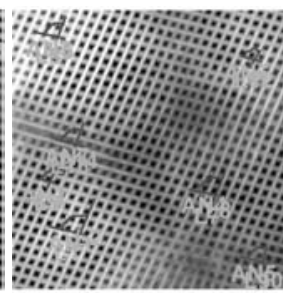

(f)
Figura 3: Análise da área 2, (a) área selecionada, (b) espectro de freqüência do ponto 1 com 12 aplicações da "spike-boost", (c) imagem recuperada (IFFT), (d) espectro de freqüência do ponto 2, (e) imagem recuperada (valor médio $\mathrm{d}_{\mathrm{hkl}}=0,89 \mathrm{~nm}$ ), (f) superposição das duas imagens recuperadas (valor médio do ângulo 98,91º).

[Figure 3: Analysis of area 2, (a) selected area on photomicrography, (b) frequency spectrum of point 1 after 12 spike boost applications, (c) recovered image (IFFT), (d) frequency spectrum of point 2, (e) recovered image (mean value $d_{h k l}=0.89 \mathrm{~nm}$ ), (e) superposition of the two recovered images (angle mean value $\left.98.91^{\circ}\right)$.]

\section{Falhas estruturais}

Foram selecionadas (Fig. 4) algumas áreas, em outras fotomicrografias, onde podem ser observados alguns defeitos de estrutura. Essas imagens foram obtidas a partir da fração grossa (2 a 0,2 $\mu \mathrm{m}$ ) de uma argila. Em geral, as melhores imagens são obtidas após 4 ou 5 aplicações da "spike-boost" na imagem original. Na primeira imagen (a) não foi medida a distância interplanar e nas duas outras os valores médios obtidos foram: $0,83 \mathrm{~nm}$ e $\mathrm{m}$ (b) e 0,72 nm em (c). Como a imagem foi obtida para uma amostra onde os óxidos de ferro

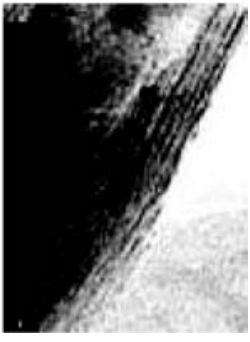

(a1)

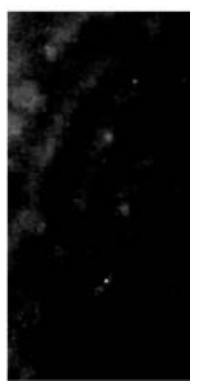

(b1)

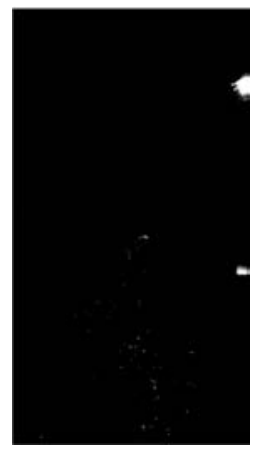

(c1)

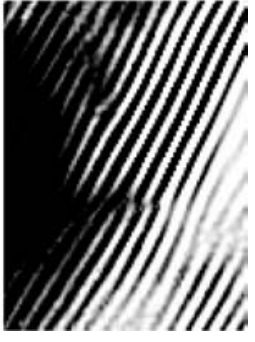

(a2)

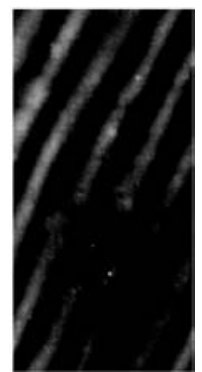

(b2)

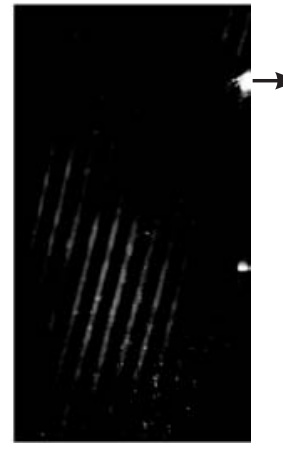

(c2)

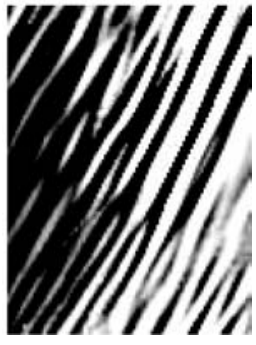

(a3)

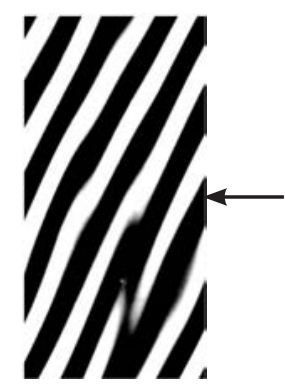

(b3)

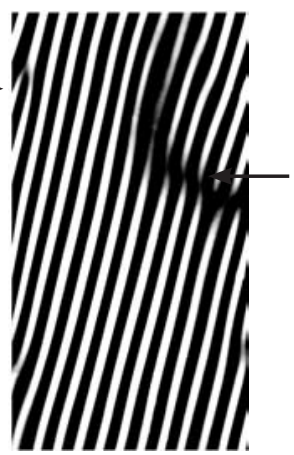

(c3)
Figura 4: Três imagens de fotomicrografias diferentes: (a1) imagem selecionada (argila), (a2) imagem recuperada (IFFT) de um ponto do espectro de freqüências e (a3) superposição de imagens recuperadas para dois pontos no espectro de freqüências; (b1) imagem selecionada (óxido de ferro), (b2) e (b3) imagens recuperadas após 3 e 7 aplicações de "spike-boost"; (c1) imagem selecionada (argila), (c2) e (c3) imagens recuperadas após 3 e 7 aplicações de "spike-boost". [Figure 4: Three images from different photomicrographs: (a1) selected image (clay), (a2) recovered image (IFFT) of one point on the frequency spectrum and (a3) superposition of recovered images for two points on the frequency spectrum; (b1) selected image (iron oxide), (b2) and (b3) recovered images after 3 and 7 spike-boost applications; (c1) selected image (clay), (c2) e (c3) recovered images after 3 and 7 spike-boost applications. ] 


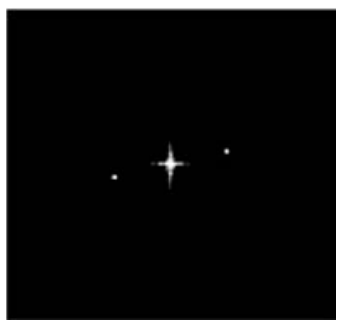

(a1)

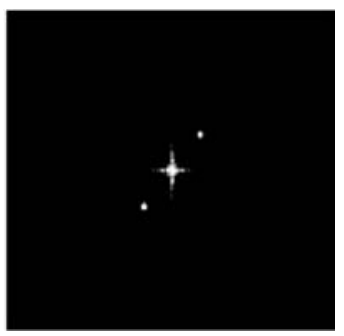

(b1)

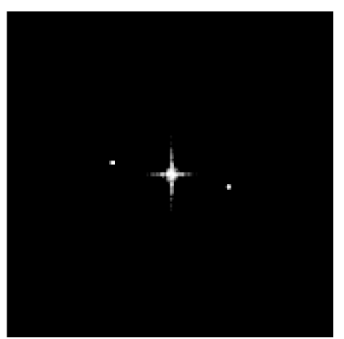

(c1)

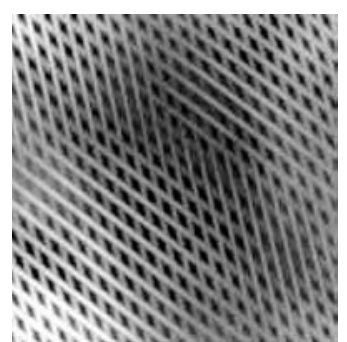

(d1)

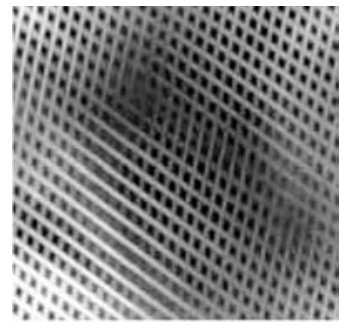

(d3)

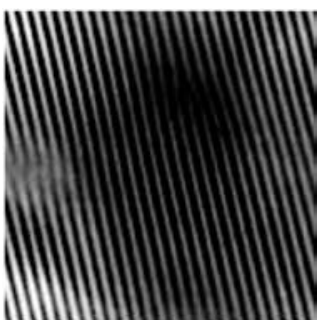

(a2)

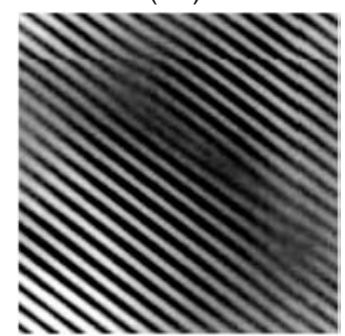

(b2)

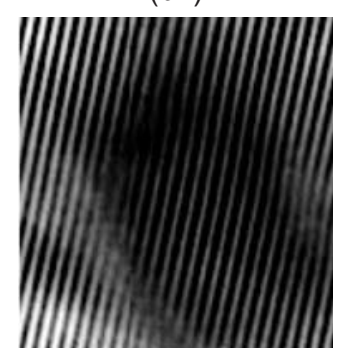

(c2)

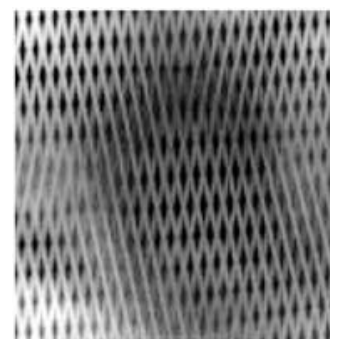

(d2)

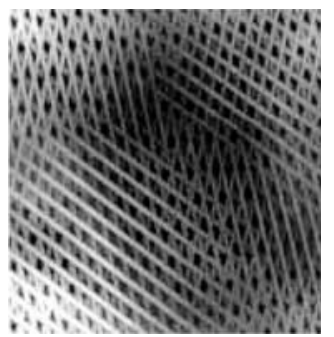

(d4)
Figura 5: Três pontos do espectro de freqüências (rede recíproca) após aplicar a ferramenta "spike boost" (a1,b1,c1) e imagens recuperadas (rede direto) para cada ponto (a2, b2,c2). Superposição das três imagens recuperadas duas a duas $(\mathrm{d} 1, \mathrm{~d} 2, \mathrm{~d} 3)$ e das três imagens (d4).

[Figure 5: Three points on the frequency spectrum (reciprocal lattice) after spike-boost tool application $(a 1, b 1, c 1)$ and recovered images (direct lattice) to each point ( $(a 2, b 2, c 2)$. Superposition of the three recovered images two-to-two $(d 1, d 2, d 3)$ and of all them $(d 4)$.] foram concentrados [7], usando separação magnética, este valor medido indica a presença de magnetita. $\mathrm{O}$ outro valor indica a presença de caulinita, que é o argilomineral principal em todas as amostras estudadas [5]. Nestas imagens também podem ser observadas deslocações em cunha e distorção da rede cristalina, na região próxima a ela. A Fig. 4 (a3) mostra uma região com vários defeitos que pode caracterizar região interestratificada de dois argilominerais, comuns em argilominerais de solos.

A Fig. 5 apresentou vários pontos no espectro de frequiências. Para obtenção desses pontos foram usadas várias ferramentas do programa: de aumento de contraste para melhor visualizar a área selecionada, FFT para obtenção do espectro de freqüências (espaço recíproco), nele também foi aplicada a ferramenta de contraste para melhor visualizar os pontos, a "spike boost" para intensificar o brilho dos pontos e a IFFT para voltar à imagem original, melhorada. A ferramenta contraste pode ser aplicada em várias situações pois ela possibilita o controle: de brilho que modifica a quantia global de luz no espectro, de contraste que muda o grau de diferença entre os componentes mais brilhantes e os mais escuros na imagem, de gama que acentua o contraste nas áreas muito claras, ou muito escuras, em uma imagem, sem alterar significativamente o contraste nas regiões de tons intermediários.

$\mathrm{O}$ espectro de freqüências melhorado (ver Fig. 2a) apresenta vários pontos que seguem um determinado padrão. Os pontos que não seguem o padrão das distribuições das freqüências no espectro são escolhidos para a aplicação da ferramenta spike-boost para aumentar o brilho dos pontos. As Figs. 5 a1, b1 e c1 mostram os três pontos selecionados, que são os nós do espaço recíproco. A transformada inversa (IFFF) destes pontos mais brilhantes fornecerá a imagem melhorada das franjas de rede do espaço direto (Figs. 5 a2, b2 e c2). A Fig. 5d mostra as combinações dois a dois e dos três conjuntos de franjas de rede, após recuperação da imagem melhorada.

Nestas imagens também foram observados defeitos de rede (deslocação em cunha) que foram destacados após a melhoria da qualidade das imagens. Das várias imagens observadas, pode-se concluir que este tipo de defeito é comum nas nano-partículas dos minerais (argilominerais, óxidos de ferro etc.) presentes nos solos.

Estas figuras permitem determinar os ângulos entre os três planos cristalográficos (hkl), caracterizados pelas franjas de rede. A combinação destas franjas mostra uma morfologia parecida com a de um tecido onde as fibras estão entrelaçadas. Deve-se tomar cuidado na análise das imagens considerando que, embora este tipo de estrutura cristalina seja regular ela pode apresentar interestratificação que pode provocar assimetria na estrutura e, também, pode ocorrer sobreposição de mais de um filossilicato na imagem sobrepondo franjas de diferentes minerais. No caso particular de argilas, onde em geral têm-se misturas argilominerais com outros minerais, o emprego de mais de uma técnica para obtenção de mais informações sobre o material é importante e recomendável. 


\section{CONCLUSÕES}

A aplicação do sistema modular de análise de imagens forneceu resultados excelentes, permitindo melhorar a qualidade das imagens, obter de forma rápida e com boa precisão informações sobre as imagens analisadas. A aplicação da FFT nas fotomicrografias de HRTEM permite observar franjas de rede com uma resolução que não é observada sem o auxílio da técnica. As várias ferramentas do sistema permitem melhorar as imagens obtidas usando microscopia eletrônica e fazer medidas importantes para o estudo de argilas, que pode ser estendido para outros materiais.

O uso desta ferramenta também permite observar defeitos de rede e obter informações sobre as estruturas de nanopartículas, como estas de ergilominerais. Acombinação das informações obtidas com dados usando outras técnicas, permitiu identificar as franjas de rede estudadas, visualizar alguns defeitos de rede e observar nanopartículas que apresentam alto grau de cristalização.

\section{AGRADECIMENTOS}

Os autores agradecem a CAPES pela bolsa de PósDoutorado (Proc. 0113/95-5, S. R. Teixeira), à FAPESP pela bolsa de iniciação científica (Proc. 01/00549-5, M. P. A. Baroni) e pelo financiamento (Proc. 99/13689-5 e 04/08957-0) do Laboratório de Microscopia da FCT/ UNESP, à Dra. A. M. O. A. Balan pela colaboração e disponibilização do laboratório.

\section{REFERÊNCIAS}

[1] R. C. Gonzalez, R. E. Woods, Processamento de Imagens Digitais, Editora Edgard Blücher Ltda, S. Paulo, Brasil (2000) 509 .

[2] M. J. Hytch, M. Gandais, Philosophical Magazine A Physics of Condensed Matter Structure Defects and Mechanical Properties 72 (1995) 619.

[3] A. B. Palotas, L. C. Rainey, C. J. Feldermann, A. F. Sarofim, J. B. VanderSande, Microscopy Res. Technique 33 (1996) 266.

[4] J. C. Barry, J. A. Alarco, J. Microscopy-Oxford 202 (2001) 495.

[5] S. R. Teixeira, J. B. Dixon, G. N. White, Texas J. Microscopy 27, 1 (1996) 11.

[6] S. R. Teixeira, J. B. Dixon, G. N. White, Texas J. Microscopy 27, 1 (1996) 11.

[7] S. R. Teixeira, J. B. Dixon, G. N. White, Texas J. Microscopy 27, 2 (1996) 43.

[8] J. B. Dixon, G. N. White, Soil Mineralogy Laboratory Manual: Agronomy 626, Soil \& Crop Sciences Dept., Texas A \& M University, College Station, TX, USA (1996) 139.

[9] A. Guinier, X-Ray Diffraction - In Crystals, Imperfect Crystals, and Amorphous Bodies, W. H. Freeman and Company, San Francisco, USA (1963) 378.

[10] F. S. Borges, Elementos de Cristalografia, Fundação Calouste Gulbenkian, Lisboa, Portugal (1980) 624.

(Rec. 25/08/2005, Ac. 11/11/2005) 